\title{
Model identification adaptive control - implementation case studies for a high manoeuvrability aircraft
}

\author{
Zsombor Öreg ${ }^{1}$, Hyo-Sang $\operatorname{Shin}^{1 *}$ and Antonios Tsourdos ${ }^{1}$
}

\begin{abstract}
With the proliferation of modular Unmanned Aerial Vehicles (UAVs) cheap and scalable control methods are needed to ensure operability. Using adaptive control it seems that these requirements could be met. In this paper applicability of parameter adaptation and control methods are demonstrated within the model identification adaptive control framework, implementing several methods and evaluating their performance. As a plant a non-linear simulation model of an F-16 aircraft is used.
\end{abstract}

\section{INTRODUCTION}

Continuous demand for increasing aircraft performance has lead to the introduction of flight control systems which now have become tremendously complex.

Typically applied control design methods (e.g. gain scheduling, dynamic inversion) require a precise dynamic model of the system, with a sophisticated aerodynamics analysis using numerical and experimental methods and flight testing well beyond the extent necessary to ensure compliance with regulations. With the proliferation of modular UAVs rapid, less expensive, scalable design methods are needed, leading to the field of self-tuning, adaptive controllers.

Adaptive controllers do not need a precise model of the plant, they can adjust for deviations in configuration as well as flight conditions. This way the robustness of the control system can be enhanced.

Design of an adaptive controller is less expensive, and an already applied controller can be easily tailored to fit the requirements of the given configuration of the aircraft.

The framework of model identification adaptive control (MIAC, also referred to as indirect adaptive control) was chosen for this paper (instead of model reference adaptive control applied more often) because of its wider applicability region (arbitrary zero-pole location may be accommodated) and due to the fact that it can be introduced in stages. Once the correct operation of model identification is ascertained, online control redesign can be applied to complete adaptation.

The aim of this paper is to investigate the applicability of MIAC for non-linear multi-input multi-output (MIMO) systems, primarily focusing on identification and parameter adaptation, which would lead to adaptive control design. For

This work was supported by BAE Systems.

${ }^{1}$ Zsombor Öreg, Hyo-Sang Shin and Antonios Tsourdos are with the Centre for Autonomous and Cyber-Physical Systems, School of Aerospace, Transport \& Manufacturing, Cranfield University, MK43 OAL, United Kingdom h.shin@cranfield.ac.uk

*Corresponding author analysis a wide range of techniques were implemented to evaluate their performance and applicability.

Applicability of identification and control methods is evaluated using a non-linear simulation model of the F-16 aircraft. The F-16 was chosen as the reference plant because it is a high-manoeuvrability, relaxed stability aircraft widely researched with an elaborate simulation model publicly available.

The F-16 is a single-place supersonic multirole fighter aircraft powered by a single turbofan engine with afterburner. The cropped-delta wing has a leading edge flap, which enables high angle of attack manoeuvres, and is adjusted automatically. Control in roll, pitch and yaw is provided by conventional flying control surfaces, ailerons, elevators and a rudder.

\section{MODEL IDENTIFICATION ADAPTIVE CONTROL FRAMEWORK}

Adaptive control provides a framework for the automatic, real-time adjustment of controllers based on the estimation of the current state of the controlled plant. Since most control techniques assume a linear plant, for the control of non-linear systems their simplified, linearised model is used at a given operating point. This simplification causes slow but a priori incalculable changes in the plant parameters. The overall goal is to track the evolution of these parameters with the accuracy within the robustness bounds of the linear controller.

A model identification adaptive controller consists of an adjustable predictor (identified system model), and adjustable controller and two adaptation mechanisms as shown in Figure 1.

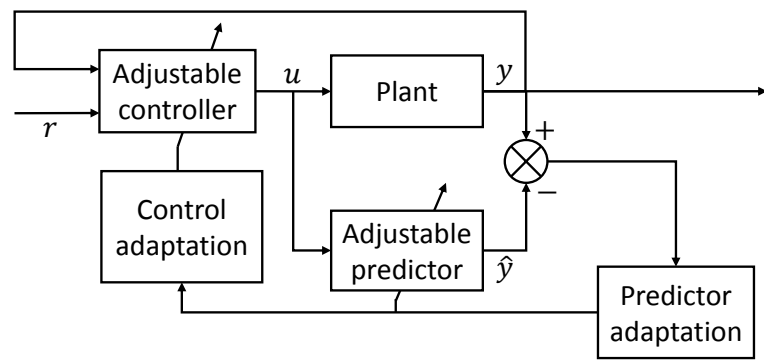

Fig. 1. Framework for model identification adaptive control (based on [1])

The adjustable predictor is the identified discrete model of the plant, and is expected to predict the plant output based on plant input sufficiently accurately. 
Parameters of the predictors are adjusted based on the output error (system identification part of the adaptive control system). Adaptation mechanisms are described in Section III.

Based on identified system dynamics, the control system can be designed. Within the scope of the paper two methods have been investigated, R-S-T and GPC, both described in Section IV.

Indirect adaptive control has already been successfully applied for flight control system design. A self-designing SISO receding horizon optimal controller has been designed as described in [2], for pitch rate control. The performance of the control design has been evaluated by simulation on the non-linear F-16/MATV model, initialised at a given trim condition. Results show that parameter adaptation and control remain effective even after effector impairment.

Comparison of performance of receding horizon controllers is done in [3], however only for the longitudinal dynamics. Superiority of adaptive RHC is demonstrated over LTI control design; however, adaptive model parameters in this case are scheduled according to flight condition dependent parameters.

For the SISO longitudinal dynamics of F-16 a linear quadratic regulator was designed in [4]. The adaptive control system is based on a nominal model, and uses a robust adaptation law to track parameter changes. The performance of the adaptive control law was compared to the classical gain scheduling control, with the adaptive having better performance.

A fault tolerant concept based on subspace system identification and model predictive control is presented in [5]. The controller designed uses a cascade controller with slidingmode control for the inner-loop, which is then identified using subspace system identification, so that a GPC can be designed for the outer loop. The system shows robustness to modelling errors and disturbances, and demonstrates sensor and effector fault tolerance.

\section{PARAMETER ADAPTATION METHODS}

An essential part of the model identification adaptive control framework is the parameter adaptation algorithm to adjust the predictor. Several approaches exist for deriving parameter adaptation algorithms [1], in this paper all methods presented are based on either the gradient technique or the least squares minimisation. The aim is to investigate whether current parameter adaptation techniques are robust and fast enough to provide a linearised model of a non-linear plant adequate for control purposes.

Although for continuous adaptation an online algorithm is needed, an attempt has been made to use an offline greybox identification method. Rather than treating the system as a black box and identifying the dynamics in a general transfer function form, grey-box identification attempts to determine the coefficients of a differential equation with a given structure. If the differential equations are the equations of motion, parameters are meaningful from an aerodynamics perspective, making identification transparent. The method applied is based on a least squares formulation and is further described in [6].

\section{A. Online, recursive algorithms}

For real-time adaptation, probably for a long period it is beneficial to use recursive algorithms since they incorporate data compression by formulation. The general structure of recursive algorithms is given by Equation (1), showing why they may be regarded as discrete integrators with the corrective term as an input.

$\left[\begin{array}{c}\text { new estimated } \\ \text { parameters }\end{array}\right]=\left[\begin{array}{c}\text { previous estimated } \\ \text { parameters }\end{array}\right]+$ $\left[\begin{array}{c}\text { adaptation } \\ \text { gain }\end{array}\right] \times\left[\begin{array}{c}\text { measurement } \\ \text { function }\end{array}\right] \times\left[\begin{array}{c}\text { prediction } \\ \text { error }\end{array}\right]$

\section{B. Gradient-based method}

The principle of gradient-based methods is to minimise error by taking subsequent steps towards the negative gradient of an error function with respect to the parameter vector [1]. Usually, the identification problem (of a transfer function or a state space) is transformed to regressor form as given by Equation (2).

$$
y(t+1)=\theta^{T} \phi(t)
$$

In Equation (2) $y(t+1)$ is the output at the next time step, $\theta$ is the parameter vector containing the system parameters and $\phi$ is the measurement vector comprising past inputs and outputs.

The minimisation problem is given by Equation (3).

$$
\min _{\hat{\theta}(t+1)} \varepsilon(t+1)=\min _{\hat{\theta}(t+1)}(y(t+1)-\hat{y}(t+1))^{2}
$$

In Equation (3) $\varepsilon(t+1)$ is the a posteriori prediction error, $\hat{y}(t+1)$ is the predicted output at the next time step based on the parameter vector at time step $t+1$.

The parameter adaptation algorithm is given by Equation (4).

$$
\hat{\theta}(t+1)=\hat{\theta}(t)+\frac{F \phi(t) \varepsilon^{0}(t+1)}{1+\phi^{T}(t) F \phi^{T}(t)}
$$

In Equation (4) $F$ is the adaptation gain matrix (a positive definite matrix) and $\varepsilon^{0}$ is the a priori prediction error, which can be calculated according to Equation (5) using the a priori prediction $\left(\hat{y}^{0}(t+1)\right)$ based on the parameter vector before adaptation $(\hat{\theta}(t))$.

$$
\varepsilon^{0}(t+1)=y(t+1)-\hat{y}^{0}(t+1)
$$

\section{Recursive prediction error method}

Gradient-based methods suffer from convergence problems around the optimum (slow convergence, overshoot, etc.), the recursive prediction error method tries to mitigate this by modifying the gradient using Newton's method. The general idea is to estimate system states together with system parameters to minimise prediction error [7]. The innovation model in state space form is given by Equations (6) and (7). 


$$
\begin{gathered}
\hat{x}(t+1, p)=A(p) \hat{x}(t, p)+B(p) u(t, p)+K(p) \varepsilon(t) \\
\hat{y}(t)=C \hat{x}(t, p)
\end{gathered}
$$

In Equations (6) and (7) $p$ is the parameter vector of elements of the matrices, $K$ is the Kalman matrix (estimated during adaptation) and $\varepsilon$ is the a priori prediction error.

The parameter adaptation formula is given by Equation (8).

$$
\hat{p}(t)=\hat{p}(t-1)+\gamma(t) R^{-1}(t) \Psi(t, \hat{p}) \Lambda^{-1} \varepsilon(t, \hat{p}(t-1))
$$

In Equation (8) $\Lambda$ is the weighting matrix (design parameter), $\gamma$ is the forgetting factor, $R$ is the weighted sample mean and $\Psi$ is the gradient matrix. Evolution of $\gamma, R$ and $\Psi$ are governed by separate formulae as given in [7].

\section{Recursive least squares}

Another method applied during the investigations was the recursive least squares algorithm. This algorithm aims at minimising the the sum of prediction errors using the current parameter estimate vector (Equation (9)) [8].

$$
\min _{\hat{\theta}(t)} \sum_{i=1}^{t}\left[y(i)-\hat{\theta}^{T}(t) \phi(i-1)\right]^{2}
$$

The parameter adaptation algorithm is summarised in Equations (10) - (13)

$$
\begin{gathered}
\hat{\theta}(t+1)=\hat{\theta}(t)+F(t+1) \\
F^{-1}(t+1)=F^{-1}(t)+\phi(t) \phi^{T}(t) \\
F(t+1)=F(t)-\frac{F(t) \phi(t) \phi^{T}(t) F(t)}{1+\phi^{T}(t) F \phi^{T}(t)} \\
\varepsilon(t+1)=\frac{y(t+1)-\theta^{T}(t) \phi(t)}{1+\phi^{T}(t) F \phi^{T}(t)}
\end{gathered}
$$

It is a necessary condition that the matrix defined by Equation (14) is invertible, which is the excitation condition.

$$
\left[\sum_{i=1}^{t} \phi(i-1) \phi^{T}(i-1)\right]
$$

From Equation (11) it can be seen that gain is decreasing constantly, which should be avoided for continuously changing parameter plants, therefore a modified equation is used (Equation (15)).

$$
F^{-1}(t+1)=\lambda_{1} F^{-1}(t)+\lambda_{2} \phi(t) \phi^{T}(t)
$$

\section{E. Directional forgetting}

Persistent excitation is difficult to satisfy for an aircraft without degrading flight performance, as a remedy directional forgetting may be utilised. Using the directional forgetting algorithm the information matrix corresponding to the parameters is decomposed to a part in the direction of the new information due to excitation and one orthogonal to it, forgetting is applied only in the direction of the new information [9]. Moreover, a threshold $(\varepsilon)$ is introduced on the amount of new information making the method more robust. The algorithm is summarised in Equations (16) - (23).

$$
\begin{gathered}
\hat{\theta}(t+1)=\hat{\theta}(t)+K(t+1)[y(t+1)-\hat{\theta}(t) \phi(t)] \\
K(t+1)=P(t+1) \phi(t)=\frac{\bar{P}(t) \phi(t)}{1+\phi^{T}(t) \bar{P}(t) \phi(t)} \\
\bar{P}(t)=P(t)+\frac{1-\mu}{\mu} \frac{\phi(t) \phi^{T}(t)}{\phi^{T}(t) R(t) \phi(t)} \text { if }|\phi(t)|>\varepsilon \\
\bar{P}(t)=P(t) \text { if }|\phi(t)|<\varepsilon \\
P(t+1)=\bar{P}(t)-\frac{\bar{P}(t) \phi(t) \phi^{T}(t) \bar{P}(t)}{1+\phi^{T}(t) \bar{P}(t) \phi(t)} \\
R(t+1)=[I-M(t+1)] R(t)+\phi(t) \phi^{T}(t) \\
M(t+1)=(1-\mu) \frac{R(t) \phi(t) \phi^{T}(t)}{\phi^{T}(t) R(t) \phi(t)} \text { if }|\phi(t)|>\varepsilon \\
M(t+1)=0 \text { if }|\phi(t)|<\varepsilon
\end{gathered}
$$

All of the above techniques have been investigated both for state space and transfer function identification when possible.

\section{CONTROL TECHNIQUES}

Two discrete control techniques have been compared, the $R-S-T$ controller and generalised predictive control.

The $R$-S-T controller was chosen because its structure properly fits the structure of the identified model using XOE (model dynamics and disturbance model). The controlled plant dynamics follow a reference model, which can be specified based on the performance standards.

Performance of $R-S-T$ is compared to the $G P C$ controller, which is optimal with respect to a given cost function providing an appropriate reference.

$\mathrm{R}-\mathrm{S}-\mathrm{T}$ control is a discrete pole placement technique decoupling tracking and regulation tasks [1]. The general architecture of an R-S-T controller is given by Equation (24).

$$
S\left(q^{-1}\right) u(t)+R\left(q^{-1}\right) y(t)=T\left(q^{-1}\right) \frac{B_{m}\left(q^{-1}\right)}{A_{m}\left(q^{-1}\right)} r(t)
$$

In Equation (24) $r$ is the reference, $q^{-1}$ is the delay operator the polynomials $A_{m}$ and $B_{m}$ provide the reference model, and $R, S$ and $T$ are the control polynomials.

Generalised predictive control intends to minimise a quadratic cost function $(J)$ penalising deviation from desired output $(e)$, deviation form steady state input $\left(u^{*}\right)$ and changes between input values $(\Delta u)$ using a predictor [10]. The quadratic cost function defined is given by Equation (25) with weighting matrices $\left(W_{y}, W_{u}, R_{u}\right)$ and horizons $\left(n_{y}\right.$ and $\left.n_{u}\right)$.

$$
J=\sum_{k=1}^{n_{y}}\left\|W_{y} e_{k}\right\|_{2}^{2}+\sum_{k=1}^{n_{u}}\left\|W_{u} u_{k}^{*}\right\|_{2}^{2}+\left\|R_{u} \Delta u_{k}\right\|_{2}^{2}
$$

Horizons were selected based on estimated settling times. 
The predictor is a transfer function given in a CARIMA form (Equation (26)).

$$
a\left(q^{-1}\right) y_{k}=b\left(q^{-1}\right) u_{k}+T\left(q^{-1}\right) \frac{\zeta_{k}}{\Delta}
$$

This way the input increments can be calculated according to Equation (27).

$$
\Delta u_{k}=\left(H^{T} H+\lambda I\right)^{-1} H^{T}\left[\vec{r}_{k+1}-P \overleftarrow{\Delta u}_{k-1}-Q \overleftarrow{y}_{k}\right]
$$

In Equation (27) the vectors $\vec{r}_{k+1}, \overleftarrow{\Delta u}_{k-1}$ and $\overleftarrow{y}_{k}$ are future references, previous input increments and previous outputs respectively, while $H, P$ and $Q$ are Toeplitz matrices constructed from model parameters and $\lambda$ contains the weights corresponding to control channels.

\section{SIMULATION MODEL}

To verify the applicability of the control methods a linearised and a non-linear model of the F-16 aircraft was used based on the data in [11] and published in [12], [13]. The model was extended to enable control development and evaluation.

The simulation model and the equations of motion therein assume that the aircraft is a constant mass rigid body, its motion can be described using Newton's equations. The mass distribution is symmetric to the longitudinal plane. The Earth is non-rotating and flat, an inertial reference system. The atmosphere is steady, variation of characteristics is described using the ISA standard atmosphere.

Using the above assumptions the full non-linear equations of motion of an aircraft can be developed, which can be linearised around trim points, according to [14]. Euler angles are used as coordinates to describe aircraft motion.

The simulation model had to be extended to enable the design of a discrete flight control system, therefore a zeroorder hold and an anti-aliasing filter have been added. Data flow diagram can be seen in Figure 2.

\section{RESULTS}

As an initial step an assessment of the identification methods was carried out, both for the linearised model and the full non-linear model. A pseudo-random binary sequence was added as a dither to input to satisfy the excitation condition.

\section{A. Grey-box identification}

Considering the benefits of an aerodynamically meaningful identification application of the grey-box identification was attempted both with the structure being the general linear equations of motion based on aeroderivatives and a general state space model. For the linearised verification model results showed good convergence even after a few seconds of simulation data, probably because of the appropriate specification of the model structure. However, when the method was applied for the off-trim input-output values of the non-linear model the algorithm did not converge, even though both a coupled and a decoupled longitudinal/lateraldirectional model was specified as a structure. The reason for the discrepancy is probably that the method estimates the disturbance model as well, thus the problem has several solutions making calculations ill-conditioned for this case. Results indicate that the method cannot be used to estimate the linearised dynamics of a highly non-linear aircraft.

\section{B. Recursive prediction error method}

For the recursive prediction error method the same dither was used to identify the system model. The identification model structure was specified as the controllability canonical state space form to reduce the number of parameters to be determined. The method has been applied for both the linear and the non-linear model. Simulation results show that if the parameter values are not estimated well enough in advance results diverge leading to an unstable estimation when the algorithm fails. The same behaviour was experienced regardless the choice of the forgetting factor limits, excitation amplitude and parameter weighting matrix. A possible explanation may be that the algorithm only guarantees convergence to a local optimum, thus if the initial estimate is not close enough to the truth values the method fails to converge. Appropriate explanation of the phenomenon required further analysis.

\section{Recursive least squares}

Since the attempts to identify system dynamics directly in a state space form have not been practically applicable due to the lack of accurate enough estimations, output error methods to identify the transfer function matrix have been adopted. Investigated techniques included filtered output error (FOE), adaptive filtered output error (AFOE) and extended filtered output error (XOE), with the last one providing the most accurate results. The main advantage of $\mathrm{XOE}$ is that it also estimates the parameters of the disturbance model given in ARMAX form, which supports effective R-S-T control design.

Assuming all states are measurable the RLS method has proved working for the identification of the transfer function matrix of the system when verified with the linear aircraft model, even if parameters are initialised as zero. The transfer function matrix then could be transformed to a state space form more conventionally used for control design for MIMO systems.

Changing the verification model to the non-linear one the parameters converge to values corresponding to an unstable system regardless of whether the total or just the off-trim input-output values are provided for the algorithm.

Since none of the above mentioned MIMO identification methods were suitable for control design, SISO models have been identified according to Table I. When providing the total input and output values for the algorithm convergence was slow, in the order of a few minutes which is impermissible for adaptive control. When using the off-trim values only convergence was achieved in a few seconds for arbitrary input values indicating that the methods need to be combined 


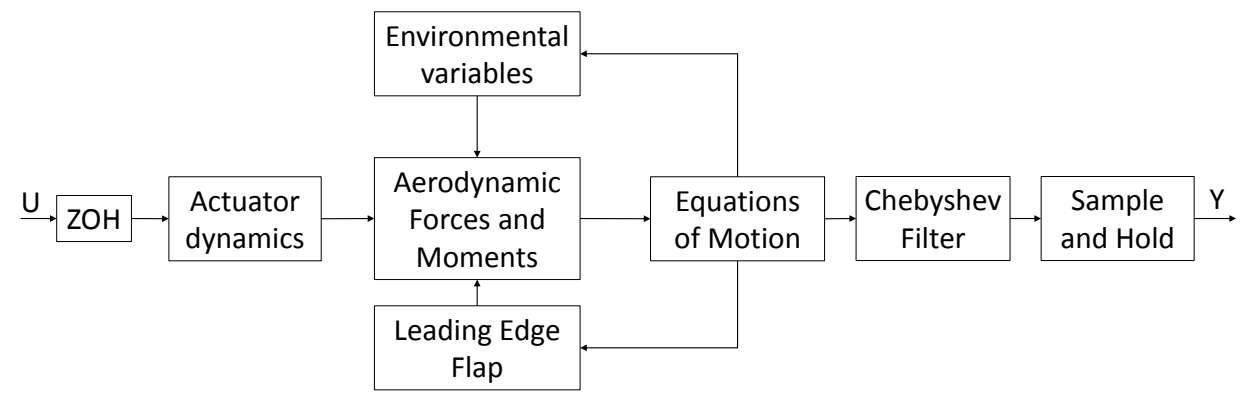

Fig. 2. Dataflow diagram of the simulation model

either with a static map of trim condition values or a separate method to estimate trim.

SISO channels were chosen by analysing the impulse response of the aircraft for each input, and correspond to traditional pairs.

TABLE I

SINGLE-INPUT-SINGLE-OUTPUT CHANNELS

\begin{tabular}{c|c|c|c}
\hline \multicolumn{2}{c|}{ Controlled plant input } & \multicolumn{2}{c}{ Controlled plant output } \\
\hline Thrust & $\tau$ & Forward velocity & $U$ \\
\hline Elevator & $\eta$ & Pitch rate & $q$ \\
\hline Aileron & $\xi$ & Roll rate & $p$ \\
\hline Rudder & $\zeta$ & Yaw rate & $r$ \\
\hline
\end{tabular}

Identification of the SISO channels using the PRBS dither showed that identification accurate enough can be achieved fast to enable adaptive control, however, identified values were highly sensitive to the initial values of the PRBS signal, especially when adaptation is performed on all channels simultaneously. Initially setting the forgetting factor dynamics parameters as $\lambda_{1}=\lambda_{2}=1$ for constant parameter estimation using standard recursive least squares a sensitivity analysis was carried out to determine which algorithm and parameters provided the best performance. Of all the forgetting factor dynamics, directional forgetting performed the best in terms of parameter adaptation rate and accuracy with a parameter selection of $\mu=0.99$ and $\varepsilon=0.001$. Using the XOE identification combined with directional forgetting for the separate channels simultaneously, based on the identified models the R-S-T and the GPC controllers could be redesigned automatically, providing the automatic control design tool without relying on any a priori knowledge of system dynamics. $\mathrm{R}-\mathrm{S}-\mathrm{T}$ reference dynamics were determined according to the Defence Standard 00-970 [15], GPC horizons were set based on settling time evaluation as $n_{y}=20$ and $n_{u}=10$. Identification and parameter adaptation can be seen in Figure 3 .

\section{Performance evaluation}

Performance of the designed controllers, both for tracking and regulation was verified using the linear model and evaluated using the non-linear model according to virtual flight tests following the Defence Standard 00-970 [15]. According to the standard the F-16 aircraft can be classified as a Class IV High manoeuvrability aeroplane. The Flight Phase chosen

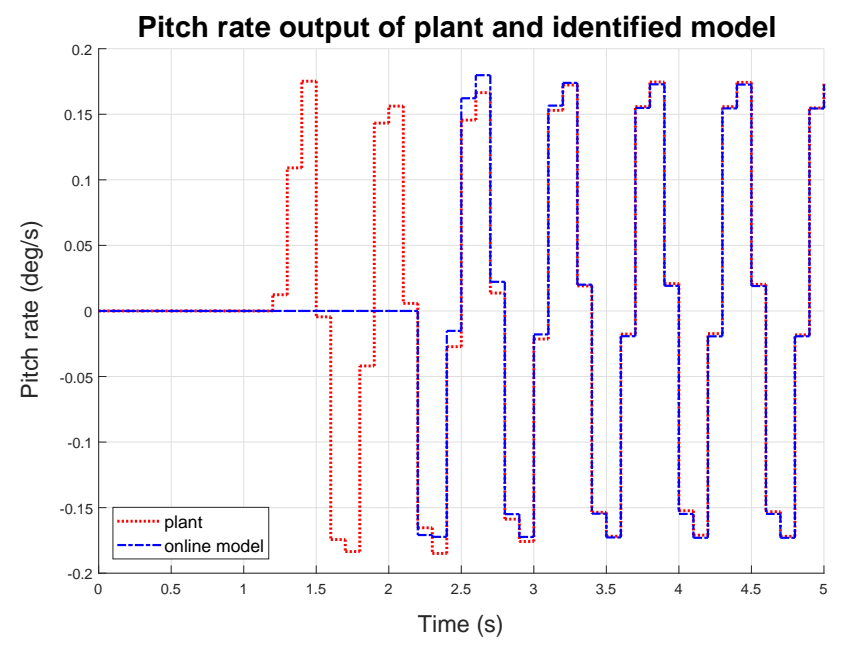

Fig. 3. Identification on the elevator $(\eta)$ - pitch rate $(p)$ channel (using XOLOE estimation technique)

is Category $\mathrm{A}$, which means a non-terminal flight phase that requires rapid manoeuvring, precision tracking or precise flight path control. SPPO, roll and spiral mode compliance is Level 1, however, for Dutch roll the compliance is only Level 2, showing that better identification for the damping of the coupled mode would be necessary to achieve better results.

Control performance of the automatically designed controllers was also evaluated qualitatively, analysing the tracking performance of a single channel while inspecting the regulation on the coupled one. The roll rate reference tracking and the yaw rate regulation are shown in Figure 4.

\section{CONCLUSION}

In this paper the applicability of model identification adaptive control for a high manoeuvrability modular UAVs was investigated, evaluating the performance on an F-16 simulation model. In search of a suitable parameter adaptation method several algorithms were implemented to identify the off-line and on-line operating point linearised dynamics of the aircraft.

Although providing satisfactory results for an off-line linearised MIMO plant, grey-box identification, recursive prediction error method and recursive least squares failed to identify linearised dynamics of the non-linear plant based 

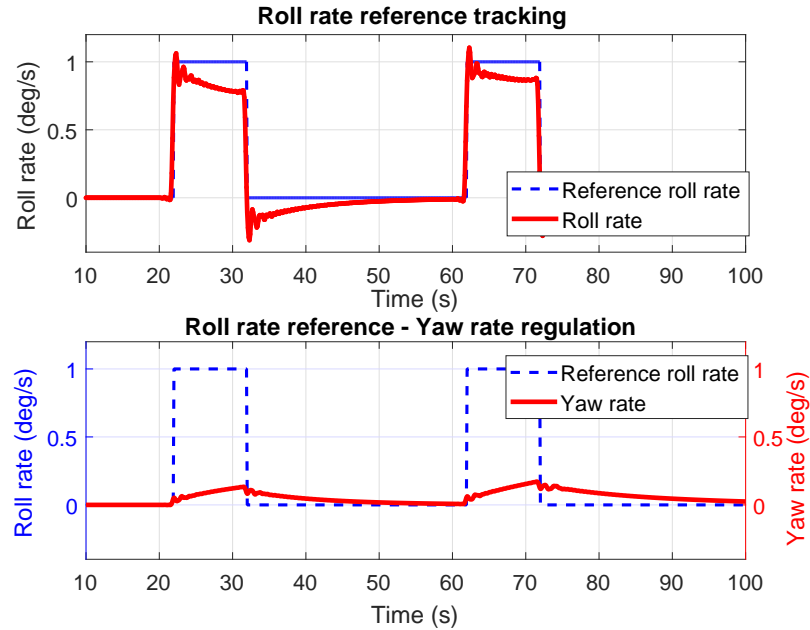

Fig. 4. Tracking for roll rate and regulation for yaw rate (using MPC control for both roll and yaw rate)

on off-trim input and output quantities thus automatic control design and adaptation was not possible. The most probable reason for that is that all methods presented are based on output error adaptation, therefore if both non-linearity and coupling between the channels is present, they cannot be distinguished based on their effect on the output. Uncertainty due to the operational point linearisation is supposed to be included in the disturbance model, while coupling effects are attempted to identified in the coupling transfer functions, however, without an appropriate filtering method no discrimination is possible resulting in significant limitations of applicability.

After decoupling dynamics to SISO channels model identification and self-tuning control redesign became possible, showing acceptable performance according to the standard. In case of a SISO channel identification both the coupling effects and the uncertainty due to non-linearity is included in the disturbance model. Effect of uncertainties and unwanted coupling effects can thus be cancelled with appropriate robust control design. Both R-S-T and GPC control designed based on the identified model showed acceptable performance and robustness.

Several open questions remain however. Since identification shows great sensitivity to the initial value of the dither sequence, dynamics of parameter convergence should be further investigated. A major drawback of the presented methods is that for the non-linear plant satisfactory results could only be obtained using the off-trim input-output values, therefore integration with a trim point estimator is necessary. Although results obtained using directional forgetting show potential to relax the excitation condition during operation, the initial discovery phase and corresponding optimal dither should also be investigated. Since it is not realistic to add a proper PRBS dither to the input, parameter convergence characteristics using initial trajectory modulation techniques have to be investigated to determine whether (combined with directional forgetting) they are sufficient to estimate the parameters with the necessary accuracy. To apply the method for the MIMO plant a method to distinguish between the effects of coupling, non-linearities and disturbance should be formulated.

\section{ACKNOWLEDGEMENTS}

The authors are grateful to Professor Rafal Zbikowski and Dr Chang-Hun Lee for their insights and expertise. The authors would like to thank the anonymous reviewers for their helpful comments.

\section{REFERENCES}

[1] I. D. Landau, R. Lozano, M. M'Saad, A. Karimi. Adaptive Control, Algorithms, Analysis and Applications. Springer-Verlag 2011

[2] D. G. Ward, R. L. Barron. A self-designing receding horizon optimal flight controller. American Control Conference, 21-23 June 1995, Seattle Washington USA, 1995.

[3] T. Keviczky, G. J. Balas. Receding Horizon Control Of An F-16 Aircraft: A Comparative Study. European Control Conference (ECC), 1-4 September 2003, Cambridge UK, 2003.

[4] Y. Huo, M. Mirmirani, P. Ioannou. Adaptive Linear Quadratic Design with Application to F-16 Fighter Aircraft. AIAA Guidance, Navigation, and Control Conference and Exhibit, 16-19 August 2004, Providence, Rhode Island USA, 2004.

[5] B. A. Siddiqui, S. El-Ferik, M. Abdelkader. Fault Tolerant Flight Control Using Sliding Modes and Subspace Identification-Based Predictive Control. IFAC-PapersOnLine, vol. 49, pp. 124-129, 2016.

[6] MathWorks. System identification Toolbox: User's Guide (R2018a), Retrieved December 30, 2018 from https://www.mathworks.com/help/ pdf_doc/ident/ident.pdf.

[7] A. E. Nordsjo, L. H. Zetterberg. A recursive prediction error algorithm for identification of certain time-varying non-linear systems. 1999 IEEE International Conference on Acoustics, Speech, and Signal Processing. Proceedings. ICASSP99 (Cat. No.99CH36258), pp. 1305-1308 vol.3, 1999.

[8] K. J. Astrom and B. Wittenmark. Adaptive Control, Addison-Wesley Publishing Company, 1989.

[9] L. Cao, H. Schwartz. A directional forgetting algorithm based on the decomposition of the information matrix. Automatica, vol. 36, pp. 1725-1731, 2000.

[10] J. A. Rossiter. Model-based Predictive Control: A Practical Approach, London, CRC Press, 2003

[11] L. T. Nguyen, M. E. Ogburn, W. P. Gilbert, K. S. Kibler, P. W. Brown, P. L. Deal. Simulator Study of Stall/Post-Stall Characteristics of a Fighter Airplane With Relaxed Longitudinal Static Stability, NASA, 1979.

[12] R. S. Russel. Non-linear F-16 Simulation using Simulink and Matlab, University of Minnesota, 2003.

[13] L. Soenneveldt. Nonlinear F-16 Model Description, Control \& Simulation Division, Faculty of Aerospace Engineering, Delft University of Technology, 2006.

[14] M. V. Cook. Flight Dynamics Principles, 2 ed. Oxford: ButterworthHeinemann- Elsevier, 2007.

[15] Ministry of Defence and Military Aviation Authority. DEF STAN 00-970. Requirements for the Design and Airworthiness of Composite Aircraft Structure, UK: Ministry of Defence and Military Aviation Authority 2014. 
2019-08-15

\title{
Model identification adaptive control - implementation case studies for a high manoeuvrability aircraft
}

\author{
Öreg, Zsombor
}

IEEE

Oreg Z, Shin H-Y, Tsourdos A. (2019) Model identification adaptive control - implementation case studies for a high manoeuvrability aircraft. In: 27th Mediterranean Conference on Control and Automation, 1-4 July 2019, Akko, Israel

https://doi.org/10.1109/MED.2019.8798513

Downloaded from Cranfield Library Services E-Repository 